\title{
L'utilisation d'outils standardisés en intervention sociale : les points de vue des intervenants, des gestionnaires et des familles sur le Protocole d'évaluation familiale en protection de la jeunesse
}

\section{The use of standardized measurements in social intervention: the perspective of child welfare workers, managers and families on the Family assessment protocol in child welfare}

\author{
Doris Chateauneuf, Catherine Arseneault et Marie-Ève Bedard Nadeau
}

Volume 48, numéro 1, 2019

URI : https://id.erudit.org/iderudit/1060004ar

DOI : https://doi.org/10.7202/1060004ar

Aller au sommaire du numéro

Éditeur(s)

Revue de Psychoéducation

ISSN

1713-1782 (imprimé)

2371-6053 (numérique)

Découvrir la revue

Citer cet article

Chateauneuf, D., Arseneault, C. \& Bedard Nadeau, M.-È. (2019). L’utilisation d'outils standardisés en intervention sociale : les points de vue des intervenants, des gestionnaires et des familles sur le Protocole d'évaluation familiale en protection de la jeunesse. Revue de psychoéducation, 48(1), 1-21. https://doi.org/10.7202/1060004ar
Résumé de l'article

Malgré la promotion et la valorisation des données probantes dans les domaines de la santé et des services sociaux, l'utilisation d'outils d'évaluation systématique en intervention sociale demeure un sujet de recherche peu exploré au Québec. Le présent article s'intéresse aux enjeux entourant l'implantation et l'utilisation d'un outil d'évaluation systématique en contexte d'intervention en protection de la jeunesse, le Protocole d'évaluation familiale (PEF). À partir des points de vue de 37 acteurs directement concernés par l'utilisation de ce protocole (intervenants, gestionnaires et familles), cette étude se penche plus spécifiquement sur les enjeux qui interviennent dans le processus d'implantation de l'outil et qui influencent son utilisation. Les résultats indiquent que le contexte organisationnel, les propriétés des questionnaires, les caractéristiques des acteurs, ainsi que les perceptions de ces derniers à l'égard de la pertinence clinique de l'outil sont les principaux facteurs soulevés par les participants pour expliquer les conditions d'implantation et le niveau d'utilisation du PEF.
Tous droits réservés @ La Corporation de la Revue Canadienne de Psycho-Éducation, 2019
Ce document est protégé par la loi sur le droit d'auteur. L’utilisation des services d’Érudit (y compris la reproduction) est assujettie à sa politique d'utilisation que vous pouvez consulter en ligne.

https://apropos.erudit.org/fr/usagers/politique-dutilisation/ 


\section{L'utilisation d'outils standardisés en intervention sociale : les points de vue des intervenants, des ges- tionnaires et des familles sur le Protocole d'évaluation familiale en protection de la jeunesse.}

\section{The use of standardized measurements in social intervention: the perspective of child welfare workers, managers and families on the Family assessment protocol in child welfare.}

\section{Chateauneuf' \\ C. Arseneault ${ }^{2}$ \\ M-E. Bedard \\ Nadeau ${ }^{3}$}

1 Centre de recherche universitaire sur les jeunes et les familles

2 Université du Québec en Outaouais

3 Université du Québec à TroisRivières

Remerciements : Les auteures tiennent à remercier l'implication de Robert Pauzé pour sa lecture attentive de l'article et pour son support tout au long du projet d'évaluation.

\section{Correspondance :}

Doris Chateauneuf

Centre de recherche universitaire sur les jeunes et les familles 2915, Avenue du Bourg-Royal, Québec (Québec), G1C 3S2

Tél. : 418 661-6951, poste 1534

doris.chateauneuf.ciussscn@ ssss.gouv.qc.ca
Résumé

Malgré la promotion et la valorisation des données probantes dans les domaines de la santé et des services sociaux, l'utilisation d'outils d'évaluation systématique en intervention sociale demeure un sujet de recherche peu exploré au Québec. Le présent article s'intéresse aux enjeux entourant l'implantation et l'utilisation d'un outil d'évaluation systématique en contexte d'intervention en protection de la jeunesse, le Protocole d'évaluation familiale (PEF). À partir des points de vue de 37 acteurs directement concernés par l'utilisation de ce protocole (intervenants, gestionnaires et familles), cette étude se penche plus spécifiquement sur les enjeux qui interviennent dans le processus d'implantation de l'outil et qui influencent son utilisation. Les résultats indiquent que le contexte organisationnel, les propriétés des questionnaires, les caractéristiques des acteurs, ainsi que les perceptions de ces derniers à l'égard de la pertinence clinique de l'outil sont les principaux facteurs soulevés par les participants pour expliquer les conditions d'implantation et le niveau d'utilisation du PEF.

Mots-clés: Protection de la jeunesse; évaluation systématique; évaluation familiale; outil d'évaluation standardisé

\section{Abstract}

Despite the promotion and development of evidence based practices in the health and social services fields, the use of standardized assessment measurements in social intervention remains a subject that has been little studied in the province of Quebec. This article focuses on at the issues surrounding the implementation and use of a standardized assessment measurements in the context of child protection interventions, the Family assessment protocol (FAP). From the perspective of 37 stakeholders directly concerned by the use of this protocol (child welfare workers, managers and families), this study examines 
the challenges involved in the tool implementation process and which influence the use of it. The results indicate that the organizational context, the questionnaires properties, the characteristics of the stakeholders, as well as the perception of the measurement clinical relevance are the main factors raised by the participants to explain the implantation conditions and the level of FAP usage.

Keywords: Child welfare; Systematic assessment; Family assessment; Standar-
dized assessment measurement

\section{Introduction}

Dans le but de développer une pratique d'évaluation des familles plus efficace et de soutenir le travail clinique des intervenants, le Centre jeunesse de Québec - Institut universitaire (CJQ-IU) (actuellement fusionné au Centre intégré universitaire de santé et de services sociaux de la Capitale nationale [CIUSSSCN]) procédait, en 2015, à l'implantation d'un protocole d'évaluation familiale (PEF). Ce protocole regroupe un ensemble de questionnaires standardisés $(n=17)$ et de questionnaires maison $(n=15)$ permettant d'évaluer les principales composantes du fonctionnement familial. Ces questionnaires ont été choisis sur la base de leurs propriétés psychométriques et de leur pertinence conceptuelle reliées aux composantes familiales à évaluer. Ils ont aussi été sélectionnés selon la facilité des parents et des jeunes à les compléter et selon leur sensibilité à détecter les changements qui pourraient survenir en cours du suivi (Pauzé, Villeneuve, Cook-Darzens, Chateauneuf et Petitpas, 2017). Ce protocole permet également de documenter différentes dimensions des jeunes vulnérables (caractéristiques biologiques, psychologiques, neuropsychologiques, langagières, sociales et scolaires) et de leurs parents (détresse psychologique, ressources sociales et économiques, histoire de maltraitance). Puisque le caractère multidimensionnel de l'abus et de la négligence envers les enfants requiert des sources multiples d'évaluations et d'interventions (Wodarski, 2015), le protocole permet de documenter différentes dimensions et répond à cette spécificité de l'intervention en protection de l'enfance.

L'implantation du PEF poursuit également différents objectifs : soutenir les intervenants dans la planification de leurs interventions; donner la parole aux jeunes et aux familles dans l'élaboration du plan d'intervention et contribuer au développement de la recherche sur les caractéristiques des jeunes et des familles bénéficiant de services en protection de la jeunesse. Le protocole s'inscrit plus globalement dans l'évaluation systématique de la pratique clinique. Kazdin (2007) définit l'évaluation « systématique » comme l'utilisation de mesures psychologiques ou d'autres mesures démontrant (ou pouvant démontrer en principe) une fiabilité et une validité, et qui offre des informations sur le fonctionnement du client qui peuvent être reproduites dans les mêmes conditions (selon ce postulat, deux cliniciens travaillant séparément devraient obtenir des résultats similaires).

Le présent article s'intéresse aux enjeux entourant l'implantation et l'utilisation d'un outil d'évaluation systématique, le PEF, en contexte d'intervention sociale en protection de l'enfance. II a pour objectif de mettre en lumière les 
points de vue des intervenants, gestionnaires et familles sur la pertinence et les enjeux d'implantation et d'utilisation du PEF en contexte d'intervention clinique. La première partie se penche sur la littérature relative à ce sujet, tandis que les sections subséquentes présentent le contexte, la méthodologie et les résultats de l'étude. Finalement, une discussion faisant état des questions et des réflexions soulevées par cette étude est proposée.

\section{Revue de littérature}

L'analyse de la littérature sur le sujet des outils d'évaluation systématique (" systematic assessment»; " standardized assessment»; "routine outcome measurement ») montre que celle-ci a surtout été développée dans le champ de l'intervention clinique en santé mentale, plus spécifiquement dans les disciplines de la psychologie et de la psychiatrie. Les enfants et les familles suivis par les services de protection de l'enfance constituent rarement la clientèle cible des études en question. Cependant, étant généralement menées auprès de psychologues, de travailleurs sociaux, de psychiatres et de thérapeutes familiaux, ces études permettent de dresser plusieurs parallèles avec le milieu de la protection de l'enfance.

Les bénéfices associés à l'utilisation d'outils d'évaluation systématique sont bien documentés. Selon Favez (2010), qui s'est intéressé aux modèles et instruments d'évaluation clinique de la famille, l'évaluation systématique appuyée par l'utilisation d'instruments de mesure " permet une prédiction significativement plus précise et donc plus utile du point de vue thérapeutique » (p. 39). De plus, les données issues des outils standardisés seraient utiles pour planifier les rencontres et organiser les entrevues (Martin, Fishman, Baxter et Ford, 2011; Valenstein et al., 2009) et pour évaluer et mesurer l'effet des interventions (Gilbody, House et Sheldon, 2002; Hatfield et Ogles, 2004, 2007; Valenstein et al., 2009). Ce type d'outil permettrait également de recueillir des informations qui autrement restent difficilement accessibles (Martin et al., 2011) et de dresser un portrait clinique détaillé de l'usager, un constat relevé tant dans le domaine de la psychiatrie (Maden, 2003), de la protection de l'enfance (Wodarski, 2015) que de l'évaluation des psychopathologies chez les enfants (Jensen Doss et Hamilton, 2005).

En outre, l'utilisation d'outils standardisés favoriserait l'implication du client dans le processus d'évaluation, dans la définition du problème et dans l'identification des cibles d'intervention (Lyon, Dorsey, Pullmann, Silbaugh-Cowdin et Berner, 2015; Martin et al., 2011). Par exemple, selon McKay et Coombs (2012), qui se sont penchés sur l'utilisation d'outils d'évaluation standardisés par des cliniciens en santé mentale, les discussions et les rétroactions autour des résultats permettent d'échanger sur les différences de perspectives. Le bilan clinique qui résulte du traitement informatisé des questionnaires servirait également d'appui au jugement clinique du thérapeute (Hatfield et Ogles, 2004; Maden, 2003). De même, plusieurs auteurs soulèvent que, lorsqu'ils sont bien utilisés et adéquatement implantés, les outils d'évaluation standardisés augmenteraient la précision et l'efficacité du processus d'évaluation et favoriseraient l'accès, pour l'usager, à un traitement et des services appropriés et adaptés à ses besoins (Garland et al., 2003; Jensen Doss et Hamilton, 2005; Lyon et al., 2015). 
Le recours à des questionnaires standardisés favoriserait l'utilisation d'un langage commun dans une équipe de travail et la collaboration multidisciplinaire, tel que le soulignent les études de Coombs, Stapley et Pirkis (2011) et de Gilbody et al., (2002), qui ont respectivement porté sur l'utilisation des outils d'évaluation standardisés dans les services en santé mentale (en Australie et en NouvelleZélande) et sur l'utilisation de mesures d'évaluation systématique chez des psychiatres (en Grande-Bretagne). En proposant une pratique d'évaluation uniformisée, ce type d'outils pallierait aux composantes subjectives du jugement clinique (Garland et al., 2003; Jenson Doss et Hamilton, 2005) et permettrait de mieux mesurer le risque et d'éviter les diagnostics trop hâtifs (Jensen Doss et Hamilton, 2005; Maden, 2003; Mash et Hunsley, 2005). Selon Rittner et Wodarski (1995), l'utilisation d'outils standardisés est d'autant plus pertinente en contexte de protection de l'enfance puisque les cliniciens (child welfare workers) sont impliqués dans des procédures décisionnelles critiques et qu'ils doivent évaluer différentes sources de données (familiales, environnementales, interactionnelles).

En dépit de l'importance accordée à l'utilisation d'outils systématiques dans les milieux d'intervention et des pressions exercées par les autorités sur les organisations pour que celles-ci démontrent l'efficacité et la qualité des services dispensés (McHugh et Barlow, 2010; Reeves, Charter et Ford, 2015), l'utilisation d'outils d'évaluation standardisés demeurerait faiblement intégrée dans les pratiques des cliniciens (Anderson et Paulosky, 2004; Bickman et al., 2000; Gilbody et al., 2002; Hatfield et Ogles, 2004). Dans un sondage réalisé auprès de 539 cliniciens travaillant auprès d'enfants et d'adolescents, seuls $23 \%$ d'entre eux mentionnaient utiliser des outils de mesures standardisés dans leurs pratiques (Bickman et al., 2000). Deux études distinctes réalisées au Royaume-Uni et aux États-Unis auprès de psychiatres indiquent que plus de la moitié d'entre eux (entre $55 \%$ et $61 \%$ ) utilisent rarement ou jamais des mesures standardisées pour l'identification et l'évaluation de la sévérité des symptômes dépressifs de leurs clients (Gilbody et al., 2002; Zimmerman et McGlinchey, 2008).

Ainsi, il semble que l'implantation d'outils standardisés comporte des enjeux bien spécifiques pour les cliniciens. Les études qui se sont penchées sur les raisons qui expliquent la disposition des cliniciens à utiliser des outils d'évaluation systématique ou, au contraire, leur propension à ne pas en utiliser, mettent en évidence différents facteurs. La formation et l'accompagnement pour les procédures d'administration de l'outil et pour l'interprétation des résultats seraient des facteurs déterminants (Hatfield et Ogles, 2004; Mellor-Clark, Cross, MacDonald et Skjulsvik, 2016; Reeves et al., 2015). Dans leur étude, Lyon et ses collègues (2015) ont mesuré les attitudes, les habiletés et les niveaux d'utilisation d'un outil standardisé en contexte d'intervention en santé mentale. Leurs résultats montrent que l'intégration des outils dans la routine des cliniciens repose en partie sur la formation et l'entraînement, mais aussi sur la promotion de l'évaluation systématique, sur la consultation et sur la mise en place de mesures de soutien à long terme. Selon Batty et ses collègues (2013), qui se sont intéressés aux enjeux des cliniciens dans l'implantation de mesures d'évaluation dans les services en santé mentale offerts aux adolescents en Grande-Bretagne, la vision des thérapeutes à propos de l'évaluation systématique et leur façon de présenter les objectifs et les méthodes d'évaluation aux clients ont également un impact sur la 
participation de ces derniers. Par exemple, si l'évaluation est présentée comme un élément normal, central et délibéré du traitement, le client serait plus susceptible de l'aborder positivement (Kazdin, 2006). D'autre part, certaines préoccupations sont aussi soulevées quant au temps exigé pour la passation et l'analyse des résultats. À ce titre, des études soulignent que l'utilisation d'outils d'évaluation systématique est parfois considérée par les intervenants comme une surcharge de travail, une exigence administrative ou une perte de temps (Boswell, Kraus, Miller, et Lambert, 2015; Garland et al., 2003; Hatfield et Ogles, 2004, 2007).

Des études soulignent également la présence de scepticisme chez certains cliniciens à l'égard des bénéfices réels associés à l'utilisation d'un outil standardisé (Garland, et al., 2003; Hatfield et Ogles 2007; Jenson-Doss et Hawley, 2010), et ce même lorsque la fidélité et la validité empiriques des outils ont été démontrées (Boswell et al., 2015). Certains cliniciens s'interrogent sur les propriétés psychométriques des outils ou manifestent des doutes à l'égard de la validité des résultats (Garland et al., 2003; Meehan, McCombes, Hatzipetrou et Catchpoole, 2006). Ils craignent que des clients éprouvent de la difficulté à comprendre les questions (Lyon et al., 2015) ou qu'ils y répondent de façon à satisfaire le thérapeute ou certains standards sociaux (désirabilité sociale) (Bilsker et Goldner, 2002; Wodarski, 2015). D'autres ont le sentiment que les outils de mesure ne sont pas adaptés à la pratique clinique et qu'ils n'arrivent pas à détecter certaines particularités individuelles ou subjectives (Gilbody et al., 2002; Lakeman, 2004) ou à établir les bons diagnostics (Martin et al., 2011).

La crainte de voir certains usagers être étiquetés aux problèmes identifiés par les outils de mesure ou encore le risque lié à la réutilisation des données et au respect de la confidentialité font également partie des préoccupations soulevées par les cliniciens et les professionnels (Batty et al., 2013; Boswell et al., 2015; Hatfield et Ogles, 2007; Maden, 2003; Martin et al., 2011). Dans une étude qualitative réalisée auprès de 50 cliniciens (services en santé mentale aux enfants et aux adolescents), dont l'objectif était d'explorer leurs attitudes à l'égard de l'utilisation d'outils d'évaluation standardisés, Martin et ses collègues (2011) constatent que plusieurs répondants, bien qu'ils reconnaissent l'utilité de ce type d'outil dans leur pratique, sont méfiants et craignent que ces derniers remplacent l'évaluation clinique ou mènent à une sous-utilisation du jugement clinique. À cet égard, les outils de mesure systématique peuvent apparaître réductionnistes aux yeux de certains intervenants (Bewick et al., 2006; Lakeman, 2004; Meehan et al., 2006). Selon les usagers interrogés dans l'étude de Moran, Kelesidi, Guglani, Davidson et Ford (2012) (parents dont les enfants bénéficient de services en santé mentale), une méthode d'évaluation basée strictement sur des questionnaires à choix multiples ne reflèterait pas toujours adéquatement la complexité de certains problèmes. Par conséquent, l'importance de diversifier les sources d'informations et les méthodes d'évaluation et de considérer l'ensemble du contexte de vie de l'usager est soulevée par plusieurs auteurs (Jenson Doss et Hamilton 2005; McKay, Coombs, Pirkis, 2012; Moran et al., 2012; Youn, Kraus et Castonguay, 2012).

Malgré son apport indéniable pour la compréhension des enjeux liés à l'implantation et à l'utilisation d'outils systématiques dans divers milieux de pratique, la littérature demeure passablement limitée en ce qui a trait aux perceptions et aux 
points de vue des acteurs concernés par l'utilisation de ce type d'outils dans le contexte spécifique de la protection de l'enfance. De plus, au Québec, les enjeux liés à l'utilisation d'outils d'évaluation systématique en intervention sociale et à leur implantation sont encore peu connus. La présente étude, en s'intéressant spécifiquement au point de vue des acteurs impliqués dans le processus d'utilisation et de passation du PEF, amène un éclairage sur les enjeux et les facteurs à considérer pour l'implantation d'outil d'évaluation systématique au sein de la protection de la jeunesse et plus globalement, de l'intervention sociale.

\section{Contexte d'implantation}

\section{Méthode}

Au CIUSSS-CN, le PEF a d'abord été implanté auprès des intervenants en protection de l'enfance qui sont en charge de faire le suivi auprès de l'enfant et de sa famille (secteur de l'« application des mesures »). À ce jour, près d'une centaine d'intervenants ont bénéficié de la formation et utilisent le PEF dans les nouveaux dossiers qui leur sont confiés. Au moment où l'intervenant convient de faire passer le PEF à une famille, il doit se rendre sur une plate-forme web et indiquer les caractéristiques de l'enfant ${ }^{1}$ (âge, sexe) et de la famille (situation conjugale et configuration familiale). Par la suite, un protocole adapté à la situation ciblée est automatiquement généré par la plateforme.

Dépendamment du profil et des compétences des parents (et le cas échéant de l'adolescent), le protocole peut être rempli de façon autonome par les répondants ou encore être complété avec le soutien et l'accompagnement de l'intervenant. Par la suite, la saisie des données colligées est informatisée et un bilan clinique faisant état de l'ensemble des résultats dans les différentes sphères évaluées est envoyé à l'intervenant responsable. À la suite de la réception du bilan clinique, l'intervenant a la responsabilité de faire une rétroaction des résultats à la famille et de discuter avec elle du et des interventions à prévoir pour pallier aux difficultés qui en émergent.

La recherche menée dans le cadre de l'implantation des protocoles visait principalement à explorer les perceptions des intervenants sociaux, des gestionnaires et des familles quant à la pertinence clinique du PEF et aux enjeux d'implantation et d'utilisation. Pour répondre à ces objectifs, 37 entretiens semidirigés ont été menés auprès de 20 intervenants, 5 gestionnaires et 12 familles. Le tableau 1 présente les détails de l'échantillon.

1. À noter que les jeunes de 12 ans et plus doivent remplir un protocole distinct de celui rempli par les figures parentales. 
Tableau 1. Échantillonnage

\begin{tabular}{lccc}
\hline & Intervenants & Gestionnaires & Familles \\
\hline Nombre potentiel & 90 & 12 & $133^{*}$ \\
Nombre réel interrogés & 20 & 5 & 12 \\
Homme & 2 & 1 & 2 \\
Femme & 18 & 4 & 10 \\
\hline
\end{tabular}

*correspond au nombre de PEF complétés à l'intérieur de la période de recrutement, soit du 1 janvier 2016 au 31 décembre 2016

\section{Recrutement}

Les intervenants et les gestionnaires ciblés pour participer à l'étude étaient tous des employés du service de l'application des mesures du CIUSSS-CN. Dans un premier temps, deux listes distinctes de participants ont été dressées: une première faisant état de tous les intervenants à l'application des mesures en service au moment de débuter la collecte de données $(n=90)$ et une deuxième, de tous les chefs d'équipe de ce même secteur $(n=12)$. Par la suite, un tirage au sort a servi à déterminer les personnes à solliciter pour participer à l'étude. Ces personnes étaient contactées par l'assistante de recherche et devaient dire si elles acceptaient ou non de participer au projet. Les entretiens avec les gestionnaires et les intervenants ont eu lieu dans leur milieu de travail, principalement dans leur bureau ou encore dans un local réservé à cette fin. La durée des entretiens réalisés variait entre 20 minutes et 90 minutes. Pour recruter les familles participantes, l'aide des intervenants sociaux a été mise à contribution. Chaque fois qu'un PEF était complété et enregistré dans le système informatique $(n=133$ pour l'année 2016), l'intervenant en charge du dossier était sollicité pour informer le ou les parents concernés de la tenue du projet de recherche et leur remettre une feuille synthèse décrivant sa nature. Lorsqu'un parent était d'accord pour participer à l'étude, les intervenants l'invitaient à signer un formulaire d'autorisation, lequel permettait par la suite à l'assistante de recherche de le contacter par téléphone. Ainsi, à la suite de la réception du formulaire d'autorisation, l'assistante de recherche entrait en contact avec le parent et validait son consentement à participer à l'étude. Si le parent était toujours d'accord à participer, son consentement verbal était enregistré et l'assistante de recherche procédait à l'entretien téléphonique. Pour minimiser les inconvénients (déplacement, temps) à la famille, les entretiens étaient réalisés par téléphone et le schéma d'entrevue était conçu de façon à ce que son utilisation ne dépasse pas 30 minutes. Une compensation financière de $20 \$$ était remise à chacune des familles participantes. Le tableau 2 indique les sujets abordés avec chacun des trois groupes de participants ainsi que le contenu des questions associé à chacun des sujets principaux. 
Tableau 2. Sujets abordés lors des entretiens selon les groupes de répondants

\begin{tabular}{llc}
\hline Sujets $\quad$ Détails et contenu des questions & $\begin{array}{c}\text { Interv } \\
n=20\end{array}$ \\
& $n=5 \quad n=12$ \\
\hline
\end{tabular}

1 Mobilisation de l'équipe

2 Utilisation et appréciation des ressources et stratégies de soutien à l'implantation

3 Perceptions en lien avec le contenu / compréhension des questions posées

4 Enjeux d'intégration du PEF dans la pratique

5 Utilité et bénéfices cliniques perçus en termes d'intervention clinique

6 Présentation et compréhension des résultats / Bilan clinique

7 Enjeux favorables et défavorables à l'implantation du PEF
- Perception de l'implantation du protocole au sein de l'équipe de travail;

- Degré de mobilisation de l'équipe par rapport au PEF.

- Niveau d'utilisation des stratégies mises en place afin de favoriser l'utilisation du PEF;

- Perceptions des répondants en lien avec l'utilité et l'appréciation de ces mesures.

- Perceptions des répondants à l'égard des questions du PEF (contenu, formulation, présence/ absence de questions embarrassantes, choquantes ou non pertinentes).

- Description par les répondants de leur rôle auprès des familles et des enjeux d'intégration du PEF à leur pratique (éléments qui facilitent / nuisent à l'utilisation du PEF).

- Perceptions des répondants à l'égard de la pertinence clinique du PEF (sa capacité à répondre aux besoins, à mettre en place des interventions prometteuses, à susciter des changements).

- Perceptions des répondants en regard de la présentation des résultats et du bilan clinique (façon dont les résultats leur ont été présentés, compréhension des résultats et du bilan clinique, sentiment qu'ils représentent bien ou non leur situation familiale).

- Description et explications par les répondants des changements de pratiques liés à l'implantation du PEF $\quad X \quad X$ et des enjeux que cela pose en termes d'interventions. 
Le processus d'analyse des données de la présente étude s'appuie sur les principes de l'analyse thématique. L'analyse thématique est une méthode d'analyse qualitative qui consiste, à partir d'une lecture attentive des données, à identifier, analyser et rapporter les "repeated patterns of meaning " (modèle répété d'unité de sens) présents dans les données (Braun \& Clarke, 2006). Fereday et MuirCochrane (2006) définissent le processus d'analyse thématique comme une « forme de reconnaissance des patterns dans les données où les thèmes émergents deviennent les catégories pour l'analyse » (p. 4). Cette démarche doit aboutir à la définition de thèmes généraux capables de rendre significatif le phénomène à l'étude (Fereday et Muir-Cochrane, 2006).

L'analyse thématique implique différentes étapes de réalisation, allant de la familiarisation aux données à la définition des thèmes identifiés. Ces étapes se présentent quelque peu différemment selon les auteurs (voir tableau 3), mais elles impliquent dans les deux cas une démarche itérative de codification et d'identification des thèmes.

\section{Tableau 3. Les étapes de réalisation de l'analyse thématique}

\begin{tabular}{lll}
\hline Braun \& Clarke (2006) & Fereday \& Muir-Cochrane (2006) \\
\hline 1 Se familiariser avec les données & 1 Développer le manuel de codes \\
2 Générer les codes initiaux & $2 \begin{array}{l}\text { Tester la fiabilité des codes } \\
3 \text { Dégager des thèmes }\end{array}$ & $3 \begin{array}{l}\text { Résumer les données et identifier les } \\
\text { thèmes initiaux }\end{array}$ \\
4 Revoir les thèmes & $4 \begin{array}{l}\text { Appliquer le modèle de codes et procéder } \\
\text { à un codage supplémentaire }\end{array}$ \\
5 Définir et nommer les thèmes & 5 Relier les codes et identifier les thèmes \\
6 Produire le rapport & 6 Corroborer et valider les thèmes codés \\
\hline
\end{tabular}

Dans le cas de la présente étude, les verbatims de toutes les entrevues réalisées ont été retranscrits. Une première lecture des entretiens a permis de dresser une version préliminaire de la grille de codification (défini comme le " code manual » par Fereday et Muir-Cochrane, 2006). Ce type de grille, développée sous forme d'arborescence (avec des codes et des sous codes), permet de faire un premier découpage du matériel et de le situer dans différentes catégories de sens. Le défi dans le cas du présent projet était de développer une grille qui puisse rassembler le contenu de tous les entretiens réalisés, autant ceux menés auprès des intervenants et des gestionnaires que ceux menés auprès des familles. Par la suite, trois entretiens (un pour chaque groupe de répondants) ont été choisis au hasard et codifiés par trois membres de l'équipe de recherche (deux chercheurs et une assistante de recherche). Cette procédure d'analyse a permis d'apporter quelques modifications à la grille de codification initiale et de s'assurer que les codes établis couvraient l'ensemble des contenus abordés par les participants. Une fois la grille validée par consensus, celle-ci a été intégrée au logiciel N'Vivo et la 
totalité des entretiens a été codée. Ces étapes de l'analyse (qui correspondent aux étapes 1 et 2 de l'analyse thématique) ont été appliquées à l'ensemble des données et ont principalement permis d'organiser le matériel et de procéder à des analyses préliminaires.

Par la suite, les données reliées à l'utilisation et à l'impact des protocoles sur la pratique clinique des intervenants ont été isolées et ont fait l'objet d'une analyse détaillée, dans le but de répondre à la question suivante : selon les différents acteurs impliqués dans l'implantation et l'utilisation des protocoles d'évaluation familiale, quels sont les enjeux cliniques et les facteurs favorables et défavorables reliés à l'utilisation et à l'implantation de ce type d'outil clinique? Cette analyse a permis de faire émerger les premiers thèmes reliés à la question posée (étape 3). Par la suite, ces thèmes ont été révisés et mis à l'épreuve (étape 4) dans le but d'en arriver à l'établissement de thèmes prédominants. Ainsi, quatre grands thèmes ont été identifiés comme étant significatifs pour répondre à la question posée et pour contenir les données présentes dans les entretiens des trois groupes interviewés (étapes 5 et 6). Selon Braun et Clarke (2006, p. 82, traduction libre), " un thème circonscrit une part importante des données en lien avec la question de recherche et représente un certain niveau de réponse ou de signification à l'intérieur de l'ensemble des données ». Les thèmes considérés significatifs sont ceux à partir desquels sont présentés et articulés les résultats.

\section{Résultats}

Les enjeux d'implantation et d'utilisation du PEF soulevés par les intervenants, les gestionnaires et les familles s'articulent autour de quatre grands thèmes : les caractéristiques des acteurs, le contexte organisationnel, les propriétés des questionnaires contenus dans le PEF, et la perception relative à la pertinence clinique de l'outil.

Tableau 4. Nombre de répondants ayant fait mention des thèmes ciblés

Thèmes

Interv Gest Fam Total

$\mathrm{n}=20 \quad \mathrm{n}=5 \quad \mathrm{n}=12 \quad \mathrm{n}=37$

\section{1- Caractéristiques des acteurs}

- Attitude de l'intervenant à l'égard du PEF (manière de présenter l'outil, valeur accordée à l'outil)

- Niveau de collaboration ou de résistance des parents (clientèle principalement non volontaire)

- Présence ou non de problématiques de santé mentale

- Niveau de scolarité des parents et capacités de compréhension

- Approche adoptée par l'intervenant auprès du parent

$\begin{array}{cccc}18 & 5 & 3 & 26 \\ 14 & 2 & -- & 16 \\ 9 & 4 & -- & 13 \\ 8 & 3 & 2 & 13 \\ -- & -- & 4 & 4\end{array}$
pour aider à la complétion. 


\section{2- Propriétés des questionnaires}

- Temps à consacrer à la complétion

- Exigence en termes de soutien et d'accompagnement

- Nombre de questionsl questionnaires à remplir

- Niveau de complexité des questions

- Complexité du processus d'analyse des résultats

- Aspect répétitif des questions

- Adaptation sur le plan culturel

\section{3- Contexte organisationnel}

- Outil qui s'ajoute aux autres obligations et approches que doivent utiliser les intervenants

- Exigences de l'outil en termes de temps (vs charge de travail)

- Pression ressentie de la part des supérieurs pour utiliser l'outil

- Intégration difficile de l'outil dans la vie et les discussions d'équipe

- Remise en question du caractère obligatoire de l'outil

\section{4- Pertinence clinique}

- Améliore la compréhension de la dynamique familiale / fonctionnement familial

- Favorise une meilleure planification de l'intervention

- Permet d'inclure la perception des familles dans l'évaluation

- Permet d'avoir accès à un nombre important d'informations

- Permet de mieux cibler les besoins de la famille

- Permet d'éviter les oublis et les omissions

\begin{tabular}{|c|c|c|}
\hline 16 & -- & 6 \\
\hline 16 & 4 & -- \\
\hline 11 & 4 & 5 \\
\hline 5 & -- & 6 \\
\hline 6 & 2 & -- \\
\hline -- & -- & 5 \\
\hline 4 & -- & -- \\
\hline 18 & 5 & -- \\
\hline 18 & 4 & -- \\
\hline 9 & 2 & -- \\
\hline 9 & -- & -- \\
\hline 6 & 2 & -- \\
\hline 17 & 5 & 7 \\
\hline 13 & 5 & 5 \\
\hline 18 & 4 & -- \\
\hline 15 & 3 & -- \\
\hline 7 & 2 & 4 \\
\hline 5 & -- & -- \\
\hline
\end{tabular}

\section{Les caractéristiques des acteurs}

La clientèle desservie par les Centres jeunesse est constituée d'enfants et de familles qui, pour la plupart, rencontrent des difficultés importantes sur les plans personnel, social et familial. De plus, l'intervention en protection de l'enfance comporte de nombreux défis, dont le contexte d'intervention généralement nonvolontaire, qui « oblige » en quelque sorte les parents à transiger avec les services de la protection de la jeunesse pour conserver la garde de leur enfant. Cette situation entraîne des défis non négligeables dans la pratique des intervenants et, parallèlement, dans l'utilisation de certains outils cliniques : "La madame a été résistante pendant tout le protocole. Elle argumentait les questions. Elle trouvait que ça n'avait pas de bon sens qu'on pose ce genre de questions-là [...] Puis, tu sais, elle est déjà contre la DPJ. Le seul élément stressant pour cette maman là, dans 
tout le questionnaire, c'était l'arrivée de la DPJ dans sa vie. Pourtant, elle a bien d'autres facteurs stressants, mais elle ne les identifiait pas " (Int-03) ${ }^{2}$. À ce titre, les intervenants indiquent qu'il est plus aisé de compléter les questionnaires auprès de parents collaboratifs qui reconnaissent leurs difficultés et qui présentent un niveau de scolarité plus élevé, puisque ces derniers démontrent moins d'opposition et comprennent mieux le sens des questions : "Bien, c'est sûr que quand les clients sont collaboratifs, quand les clients ne démontrent pas tant de résistance, quand ils sont volontaires, quand ils sont aussi relativement éduqués, c'est plus facile " (Int-02). À l'inverse, des familles réfractaires à recevoir des services ou des parents présentant des troubles de santé mentale ou des difficultés de compréhension sont des facteurs qui, selon les intervenants, compliquent l'introduction et la passation du PEF : « On a beaucoup d'illettrés fonctionnels dans notre clientèle... beaucoup de gens démunis qui ont de la difficulté à lire ou qui sont incapables de lire. [...] Je te dirais que l'outil n'est pas nécessairement adapté pour tout le monde » (Int20). Ainsi, selon le contexte et la capacité des parents à répondre aux questions, l'intervenant peut se voir dans l'obligation d'accompagner étroitement le parent dans la complétion des questionnaires, ce qui peut agir comme facteur défavorable en raison du temps que cela exige: " Des fois, il y a de l'accompagnement spécifique qui doit être fait. Ça, je reconnais que ça peut être un peu plus laborieux et nécessiter davantage à l'intervenant » (Gest-03).

Par contre, le soutien des intervenants auprès des familles ayant rencontré des difficultés dans la complétion du PEF s'avère très apprécié de la part des parents : " Des fois, c'était plus ou moins embêtant à répondre, elle [intervenante] m'a accompagnée là-dedans pour, justement, être sûre que je comprenne le sens de la question pour donner une bonne information de ma part [...] Je pense que c'est important d'accompagner le parent pour remplir le questionnaire " (Fam-06). Bien qu'il soit demandé aux intervenants d'effectuer systématiquement un PEF à chaque nouveau dossier, plusieurs mentionnent que certaines conditions liées aux caractéristiques des usagers empêchent l'utilisation systématique du PEF dans tous les dossiers :

On ne peut pas le faire d'emblée exactement comme les gens [en référence à l'administration] voudraient que ça soit fait. À cause de la clientèle, à cause des problématiques, à cause de la collaboration. II y a plein de facteurs (Int-19).

Je regarde un peu la collaboration aussi de la famille. Il y a des gens avec qui, quand la collaboration n'est pas top, ou quand il y a peut-être des fragilités de santé mentale, quand il y a beaucoup de méfiance... Il y a un type de clientèle que je fais : "ok, non » (Int-05).

2. Pour simplifier la présentation des résultats, les premières lettres entre parenthèses réfèrent au type de répondant : "Int » réfère à un entretien avec un intervenant, "Gest » à un gestionnaire et «Fam » à une famille. 
Les conditions de passation du PEF dépendent donc en partie des caractéristiques des usagers, mais elles dépendent aussi de celles des intervenants. Bien que ces derniers aient développé différentes manières de procéder pour introduire et présenter le PEF aux familles, leur attitude à l'égard du PEF et leur façon de le présenter aux usagers influencent la réussite du processus. À ce titre, les gestionnaires insistent sur le fait que les intervenants doivent croire au PEF et à son utilité afin de l'introduire positivement aux familles: "Comme je dis, il faut vraiment, il faut que l'intervenant soit vendeur dans son "pitch" de vente avec le client. II faut que l'intervenant y croie » (Gest-01). Par ailleurs, la majorité des intervenants conviennent que lorsque le PEF est bien présenté aux familles ciblées, celles-ci acceptent généralement d'y prendre part. De plus, l'ensemble des parents interviewés affirme avoir accepté de remplir les questionnaires du PEF sans hésitation et sans crainte en raison de l'expérience de l'intervenant, des explications fournies en lien avec l'utilité de cet outil ou en regard des normes de confidentialité : " De la manière qu'elle m'a expliqué le principe et tout. Elle dit : "Ça, ça va nous donner un bilan pour pouvoir vraiment cibler, vous apporter le soutien qui vous correspond. C'est comme faire un plan d'intervention ». Elle dit: "Je vais individualiser ». [...] C'est pour ça que moi j'ai donné mon accord tout de suite " (Fam-06). Plusieurs intervenants présentent également le PEF comme un outil permettant aux familles de faire connaître leurs perceptions quant à leur situation et aux intervenants d'évaluer adéquatement les besoins des familles : « Je pense que c'est important en protection de la jeunesse que les gens aient une place pour exprimer comment ils perçoivent leur vie et leur situation, parce que ce n'est quand même pas rien quand la DPJ débarque dans ta vie [...] Nous, après, ça nous donne des pistes d'intervention avec eux » (Int-07).

\section{Le contexte organisationnel}

Le contexte de travail des intervenants est aussi soulevé pour expliquer certains des enjeux rencontrés dans l'implantation et l'utilisation du PEF. Dans un premier temps, la charge de travail des intervenants est souvent nommée comme un frein à l'utilisation du PEF. À cet effet, la plupart des intervenants mentionnent avoir peu de temps pour la passation du PEF en raison des autres tâches desquelles ils doivent s'acquitter (gestion de crises, rédaction de rapports, comparutions au tribunal, temps disponible limité, dossiers prioritaires, etc.) :

Parallèlement à ça [en référence au PEF], moi j'ai quand même des choses à aborder avec la cliente, mais là, si je le prends sur le protocole, bien je n'ai pas assez de temps pour ce que moi j'ai besoin de lui jaser... tout s'allonge au niveau du temps. Ça fait que l'horaire devient chargé, ça devient plus complexe (Int-03).

Avec la charge de travail que l'on a, c'est difficile de le faire... Puis, l'idéal, ce serait de le passer dans tous nos dossiers puis moi je n'y arrive pas... Je ne vois pas comment je peux arriver à tous les passer (Int-01). 
La gestion des urgences ainsi que l'obligation d'utiliser dans leur pratique d'autres approches viennent également restreindre l'utilisation du PEF : "Tu as le plan d'intervention, tu as SOCEN ${ }^{3}$ pour nos jeunes qui sont placés à majorité, tu as les tout-petits, bien la démarche de clarification de projet de vie... Regarde, tout est à faire. [...] C'est ce qui fait qu'un moment donné, quand tu additionnes tout ça, tu en as trop » (Int-01). Plusieurs déplorent également que l'administration ajoute le PEF à leur liste de tâches, sans pour autant réviser l'ensemble de leurs obligations et responsabilités : "Notre travail a beaucoup évolué au cours des dernières années, puis on nous demande de plus en plus de choses, mais ils en n'enlèvent pas à l'autre bout » (Int-20).

Par ailleurs, le « caractère obligatoire » du PEF suscite des réactions chez certains intervenants qui considèrent que l'organisation devrait leur laisser le choix des outils à utiliser et des moments de leur passation. Plutôt que d'utiliser le PEF avec chaque nouvelle famille, plusieurs intervenants jugent plus pertinent d'évaluer la situation et les caractéristiques des membres de la famille afin de déterminer s'ils sont aptes compléter l'outil : "Tu me formes, je m'engage à les appliquer, mais fais confiance à mon jugement clinique pour les appliquer aux bons moments » (Int-04). De plus, selon certains intervenants, le début de suivi n'est pas le moment le plus propice pour l'utilisation du PEF : « Là, ce n'est pas réaliste de penser qu'il faut que je crée mon lien avec la famille, que je fasse le protocole et que je fasse un plan d'intervention initial » (Int-10). À cet égard, les intervenants pensent qu'ils devraient eux-mêmes pouvoir cibler le moment le plus opportun pour la complétion du protocole.

\section{Les propriétés et caractéristiques des questionnaires}

Le protocole lui-même et les questionnaires qui le composent ont été peu soulevés par les répondants comme enjeu à son utilisation. Les familles interrogées, principales concernées puisque ce sont elles qui sont appelées à remplir les questionnaires, considèrent que les questions du PEF sont généralement faciles à comprendre : "II y a des questions qui étaient un peu semblables, un peu, mais c'était quand même bien expliqué. C'était bien, ç'a été facile » (Fam-03). Quelques défis reliés au format des questions posées ont tout de même été mentionnés, dont la formulation plus complexe de certaines questions, la présence de questions répétitives ou encore l'aspect restrictif des choix de réponses :

Je dirais que c'est beaucoup de réponses "totalement en désaccord », "un peu en désaccord ». Je trouve que la palette d'échelle... je trouve ça compliqué des fois. La ligne est un peu mince entre chaque. (Fam-01)

Il y avait des questions qui se répétaient. Je répondais quelque chose à certaines questions, la question d'après ou la question plus loin, c’était un peu la même (Fam-03)

3. L'abréviation « SOCEN » réfère à l'approche « S'occuper des enfants ». Cette approche vise plus spécifiquement les enfants placés en famille d'accueil. 
De plus, les questions du PEF identifient les forces des familles. Puisqu'elles ont souvent l'habitude de discuter des aspects négatifs, surtout en contexte de protection de l'enfance où la prise en charge de l'enfant est souvent associée aux problèmes des parents ou à des lacunes familiales, les familles apprécient discuter des aspects positifs de leur situation : "Ce qui est le fun aussi, ce que j'ai bien aimé, ça ne dit pas juste ce qui ne va pas. Ça dit aussi ce qui va bien. C'est un peu encourageant »(Fam-06).

Cependant, la longueur du processus et l'investissement que requiert le PEF peuvent constituer des conditions défavorables à son utilisation. En effet, les intervenants mentionnent que les questionnaires comportent beaucoup d'items qui nécessitent une plus longue réflexion, ce qui allonge le temps de passation : « Puis, les points négatifs, bien, c'est le temps de remplir les questionnaires [rires], je l'ai dit plusieurs fois là... [...] C'est des questions qu'il faut que tu réfléchisses. Il y a des questions que tu dois prendre le temps. C'est un bon investissement " (Int12). De plus, les étapes à franchir pour compléter l'ensemble du PEF (passation, accompagnement, lecture du bilan clinique, rétroaction auprès de la famille) sont nombreuses et requièrent, selon les intervenants, un investissement important en termes de temps, autant pour eux que pour les familles.

\section{La pertinence clinique}

Malgré les défis d'implantation et d'utilisation du PEF, sa pertinence comme outil clinique est largement reconnue. En effet, tant les intervenants, les gestionnaires que les familles s'entendent sur la plus-value du PEF et de ses retombées positives sur l'intervention clinique. Selon plusieurs intervenants, le PEF améliore leur compréhension de la dynamique familiale et permet de bonifier leur analyse de la situation familiale :

Ça vient vraiment nous donner beaucoup d'infos sur la situation de chaque personne de la famille, ça peut nous amener à dresser un portrait là quand même très précis de la situation familiale et des personnes qui sont concernées (Int-12).

Moi, je vois une différence dans ma perception que j'ai de la famille. [...] on dirait que j'ai plus une vue d'ensemble de la famille maintenant. [...]. Même des fois, si je ne l'utilise pas, je vais quand même adopter les concepts et ça met du vocabulaire sur des rapports, ça met du vocabulaire sur... pour expliquer les choses. (Int-08)

Plusieurs intervenants et gestionnaires affirment également que le PEF favorise une meilleure planification de l'intervention auprès des familles, dans la mesure où il permet de mieux cibler les besoins des familles, de prioriser les interventions et d'aller au-delà des motifs de compromission :

Je trouve ça vraiment pratique pour ça, parce que ça établit des priorités en fonction des perceptions des familles. (Int-19) 
Ça aiguise un peu plus notre..., nos questions, notre façon d'intervenir. On voit plus large que juste les problèmes inscrits au niveau de nos éléments de compromission. (Int-12)

D'un point de vue professionnel, le PEF permet également d'uniformiser la pratique entre les intervenants et fournit une ligne de conduite. Par exemple, l'utilisation du PEF permet d'éviter les oublis et les omissions, puisque chaque question est posée systématiquement. Ainsi, le PEF fournit un cadre d'évaluation qui réfère moins à la subjectivité de l'intervenant et qui permet d'appuyer l'évaluation de la situation familiale sur des concepts partagés : " Ça nous donne une base plus solide puis ça nous permet vraiment de faire le tour puis de mettre des mots. De mettre des mots sur des concepts puis des choses qu'on observait mais qui avant étaient juste des observations » (Int-17).

D'autre part, les intervenants et les gestionnaires soulignent que le PEF s'avère être un bon levier d'intervention pour l'adhésion des familles. En travaillant avec les familles à partir de leurs perceptions et de leurs points de vue, la collaboration serait facilitée : "Le protocole c'est un outil qui est agréable à utiliser dans cette optique-là où on peut comparer notre opinion clinique avec la perception des gens, trouver des points de travail communs entre ce que les gens veulent changer. C'est un levier d'intervention aussi, dans le fond... pour travailler avec nos clients ». (Int-07)

Le PEF permet aux familles de se prononcer sur leur propre situation et ainsi de se sentir impliquées dans le processus d'évaluation: "Moi, je pense que les familles se sentent plus impliquées quand on fait plus attention à leurs perceptions. [...] En tenant compte de leurs perceptions et des choses qui, eux, les dérangent, ça les implique davantage » (Int-19). Les familles interrogées sont généralement en accord avec ces propos. En plus de leur fournir une occasion de partager leurs perceptions, le PEF a permis à certaines d'entre elles de réfléchir à leur situation et de mieux comprendre leur fonctionnement familial : "C'est un bel outil aussi. Ça m'a appris quand même beaucoup de choses, même par rapport à moi. J'ai appris à me connaître un petit peu mieux avec ça. Non, c'est vraiment positif comme expérience " (Fam-02).

Le protocole encourage également certains parents à se dévoiler davantage, puisqu'il est parfois moins embarrassant ou compromettant de nommer certaines choses sur papier que d'en discuter directement avec l'intervenant : " $D e$ pouvoir nommer des choses parce que, des fois, en mots, les gens n'oseront pas, alors que par écrit c'est un médium qu'ils vont se permettre de nommer " (Int-11). Inversement, le PEF permet aux intervenants d'obtenir des informations sur des sujets qu'ils n'auraient peut-être pas abordés d'emblée en contexte clinique : "Ça vient contourner un peu, je te dirais, les limites de l'intervenant. Relativement à des aspects pour lesquels il est peut-être moins à l'aise d'aller explorer, que ce soit la sexualité, le suicide, la toxicomanie, etc. » (Gest-03).

Somme toute, la plupart des intervenants, des gestionnaires et des familles sont d'avis que le PEF entraîne des impacts positifs à l'intérieur des familles. En impliquant celles-ci dans le processus, en leur permettant de réfléchir à leur 
situation et de mieux comprendre leur fonctionnement familial, le PEF génère plusieurs bénéfices dans le suivi et les interventions qui leur sont destinées.

\section{Discussion}

Solliciter la participation des parents pour la complétion de questionnaires peut constituer un défi de taille en protection de l'enfance, considérant la réticence de certains parents à collaborer avec les intervenants, mais aussi en raison des différents problèmes et des défis que rencontrent les parents. Plusieurs des difficultés mentionnées par les intervenants relativement à l'utilisation du PEF demeurent liées au contexte d'intervention et aux caractéristiques de la clientèle en protection de la jeunesse, une clientèle souvent non volontaire, qui fait face à de nombreux problèmes sur les plans personnel, familial ou social et pour quil l'assiduité et l'implication dans le suivi psychosocial est parfois une difficulté supplémentaire. Par ailleurs, lorsque l'intervenant réussit à développer un lien de confiance avec la famille, la collaboration est plus facile et l'expérience reliée à la passation du PEF est considérée positive par les parents interrogés dans la présente étude. Considérant que sur le plan clinique, la participation de l'usager permet son intégration dans le processus d'évaluation (Lyon et al., 2015; McKay et Coombs, 2012; Wodarski, 2015) cette position de la part des familles interrogées constitue un levier non-négligeable pour leur adhésion au suivi psychosocial.

Dans la présente étude, bien que la plupart des intervenants s'entendent sur la valeur clinique de l'outil, les avis divergent en ce qui concerne le réalisme et la facilité du contexte d'utilisation. L'outil est considéré exigeant en termes d'investissement et d'accompagnement et les intervenants considèrent manquer de temps pour l'intégrer adéquatement à leur pratique. Par ailleurs, bien que le soutien offert aux familles dans la complétion du PEF soit identifié par ces dernières comme une condition favorable et aidante, il est plutôt perçu comme une condition défavorable par les intervenants en raison du temps que cela exige. Une situation qui peut sans doute s'expliquer par le fait que l'intervenant porte la responsabilité non seulement de la passation de l'outil, mais également de l'intégration des résultats (bilan) et du suivi auprès de la famille. Les études de Martin et al. (2011) et de Meehan et al. (2011) dans les domaines de la santé mentale et de la psychiatrie montrent que les intervenants présentent des positions partagées et des attitudes variables à l'égard de l'utilisation d'outils d'évaluation standardisés et qu'ils rapportent des avantages et des inconvénients de manière équivalente. La présente étude révèle que les familles sont globabement satisfaites de la passation du PEF, alors que les intervenants sont plus enclins à souligner les difficultés et les conditions d'utilisation défavorables. Ces résultats sont comparables à ceux de McKay et al. (2012) qui constatent que, dans le domaine de la santé mentale, les clients tendent à percevoir davantage les bénéfices des mesures standardisées tandis que les cliniciens se montrent moins enthousiastes.

Contrairement à certaines études (Bilsker et Goldner, 2002; Garland et al., 2003; Meehan, McCombes, Hatzipetrou et Catchpoole, 2006) qui mentionnent que les intervenants doutent ou remettent en question la validité des outils standardisés, les participants interrogés dans la présente étude n'ont pas soulevé cet enjeu. En effet, les résultats montrent que tant les intervenants que les gestionnaires 
reconnaissent la valeur clinique de l'outil et ne remettent pas en question les propriétés psychométriques des questionnaires ou leur capacité à dégager des informations pertinentes et valides en termes d'intervention.

Par contre, à l'instar des intervenants interrogés dans l'étude de Martin et al. (2011), plusieurs intervenants de notre étude s'interrogent sur la conjugaison entre l'utilisation d'outils standardisés et la place accordée au jugement clinique. Par exemple, le caractère obligatoire du PEF suscite des réactions de la part des intervenants qui soutiennent que le protocole n'est pas adapté à certaines familles ou profils de parents et qu'en ce sens, ils devraient pouvoir juger eux-mêmes avec qui et quand il est pertinent d'utiliser le PEF. À cet effet, les études de Batty et al. (2013) et de McKay et Coombs (2012) rappellent qu'il est important que les cliniciens comprennent la pertinence clinique de l'outil et n'aient pas le sentiment que la passation d'outils standardisés est simplement une commande administrative.

\section{Forces et limites de l'étude}

Le fait de s'intéresser aux positions de trois groupes d'acteurs différents impliqués dans l'implantation ou la passation du protocole (gestionnaires, intervenants et familles) permet de capter une multiplicité de points de vue et de proposer une analyse plus complète des différents enjeux rencontrés par chacun des groupes. De plus, la participation des familles suivies en protection de l'enfance à un projet de recherche constitue toujours un défi méthodologique de taille qui, dans le cas de cette étude, a été relevé. Par contre, certaines limites sont observées. Tout d'abord, notons que la collecte de données a été réalisée en un seul temps de mesure et qu'il n'a pas été possible de recueillir les perceptions des acteurs à différents moments et, ainsi, de pouvoir constater l'évolution des perceptions à l'égard de l'implantation et de l'utilisation du PEF. À un autre niveau, mentionnons que les familles qui ont accepté de participer à l'étude sont sans doute celles qui avaient déjà un lien positif avec leur intervenant, puisque c'est ce dernier qui établissait le contact initial avec la famille (dans le cadre du recrutement). Cette situation a pu faire en sorte que les familles participantes étaient des familles déjà relativement bien impliquées dans leur suivi psychosocial.

\section{Implications pour la pratique}

L'implantation et l'utilisation d'outils d'évaluation standardisés dans les milieux d'intervention psychosociale exigent des investissements importants de la part des organisations concernées, tant en regard des ressources humaines que financières. L'intégration dans la pratique de ce type d'outil nécessite un engagement explicite de l'organisation qui se traduit par l'accès à des formations et à des mesures de soutien (Hall et al., 2014; Lyon et al. 2005), mais aussi par une révision de l'ensemble des tâches des intervenants à la suite de l'introduction d'un outil. Si la dimension financière constitue un facteur peu abordé dans la présente étude, elle représente néanmoins un point névralgique du processus d'implantation (Batty et al., 2013; Boswell et al., 2015). Dans un contexte de coupures budgétaires et de rationalisation des services, cet enjeu mérite d'être réfléchi et planifié par les organisations. 
Du côté des intervenants, le déf est d'arriver à trouver un équilibre entre l'utilisation d'outils standardisés et leur jugement clinique, donc d'arriver à considérer les données scientifiquement produites tout en conservant une part d'intuition (Alain et Rousseau, 2014). Les intervenants, d'abord préoccupés par la relation d'aide et par la subjectivité des acteurs, peinent parfois à considérer les outils et les questionnaires standardisés comme un allié dans leur pratique clinique. Le compromis professionnel associé à cette recherche d'équilibre constitue sans doute lui aussi un enjeu d'implantation qui devrait être examiné par les organisations et administrateurs.

\section{Références}

Alain, M. et Rousseau, M. (2014). Recourir aux données probantes dans l'intervention psychosociale : passage obligé ou effet de mode? La pratique en mouvement, 7,14-15.

Anderson, D. A. et Paulosky, C. A. (2004). A survey of the use of assessment instruments by eating disorder professionals in clinical practice. Eating and Weight Disorders, 9, 238-241. http://dx.doi.org/10.1007/ BF03325075

Batty, M. J., Moldavsky, M., Foroushani, P. S., Pass, S., Marriott, M., Sayal, K. et Hollis, C. (2013). Implementing routine outcome measures in child and adolescent mental health services: From present to future practice. Child and Adolescent Mental Health, 18(2), 82-87. http://dx.doi.org/ 10.1111/ j.14753588.2012.00658.x

Bewick, B. M., Trusler, K., Mullin, T., Grant, S. et Mothersole, G. (2006). Routine outcome measurement completion rates of the CORE-OM in primary care psychological therapies and counselling. Counselling and Psychotherapy Research, 6(1), 33-40. http://dx.doi. org/10.1080/14733140600581432

Bickman, L., Rosof-Williams, J., Salzer, M. S., Summerfelt, W. T., Wilson, S. J. et Karver, M. S. (2000). What information do clinicians value for monitoring adolescent client progress and outcomes? Professional Psychology: Research and Practice, 31(1), 70-74. http://dx.doi.org/10.1037/0735-7028. 31.1.70
Boswell, J. F., Kraus, D. R., Miller, S. D. et Lambert, M. J. (2015). Implementing routine outcome monitoring in clinical practice: Benefits, challenges, and solutions. Psychotherapy Research, 25(1), 6-19.

Bilsker, D. et Goldner, E. M. (2002). Routine outcome measurement by mental healthcare providers: Is it worth. The Lancet, 360(9346), 1689-1690. http://dx.doi. org/10.1016/S0140-6736(02)11610-2

Braun, V. et Clarke, V. (2006). Using thematic analysis in psychology. Qualitative Research in Psychology, 3(2), 77-101. http://dx.doi.org/10.1191/1478088706 qp063oa

Coombs, T., Stapley, K. et Pirkis, J. (2011). The multiple uses of routine mental health outcome measures in Australia and New Zealand: Experiences from the field. Australasian Psychiatry, 19(3), 247-253. http://dx.doi.org/10.3109/1039 8562.2011.562507

Favez, N. (2010). L'examen clinique de la famille: Modèles et instruments d'évaluation. Wavre, Belgique: Édition Mardaga.

Fereday, J. et Muir-Cochrane, E. (2006) Demonstrating rigor using thematic analysis: A hybrid approach of inductive and deductive coding and theme development. International Journal of Qualitative Methods, 5(1), 80-92.

Garland, A. F., Kruse, M. et Aarons, G. A. (2003). Clinicians and outcome measurement: What's the use? The Journal of Behavioral Health Services et Research, 30, 393-405. http://dx.doi. org/10.1007/ BF02287427 
Gilbody, S. M., House, A. O., et Sheldon, T. A. (2002). Psychiatrists in the UK do not use outcomes measures: National survey. British Journal of Psychiatry, 180, 101-103. http://dx.doi.org/10.1192/ bjp.180.2.101

Hall, C. L., Moldavsky, M., Taylor, J., Sayal, K., Marriott, M., Batty, M. J., ... et Hollis, C. (2014). Implementation of routine outcome measurement in child and adolescent mental health services in the United Kingdom: a critical perspective. European Child and Adolescent Psychiatry, 23(4), 239-242.

Hatfield, D. R. et Ogles, B. M. (2004). The Use of Outcome Measures by Psychologists in Clinical Practice. Professional Psychology: Research and Practice, 35(5), 485-491. http://dx.doi. org/10.1037/0735-7028.35.5.485

Hatfield, D. R. et Ogles, B. M. (2007). Why some clinicians use outcome measures and others do not. Administration and Policy in Mental Health and Mental Health Services Research, 34(3), 283291. http://dx.doi.org/10.1007/s10488006-0110-y

Jensen-Doss, A. et Hamilton, J. (2005). Evidence-based diagnosis: Incorporating diagnostic instruments into clinical practice. Journal of the American Academy of Child et Adolescent Psychiatry, 44(9), 947-952.

Jensen-Doss, A. et Hawley, K. M. (2010). Understanding barriers to evidencebased assessment: Clinician attitudes toward standardized assessment tools. Journal of Clinical Child and Adolescent Psychology, 39(6), 885-896. http:// dx.doi.org/10.1080/15374416.2010.517 169

Kazdin, A. E. (2006). Assessment and evaluation in clinical practice. In C. D. Goodheart,A. E. Kazdin et R. J. Sternberg (Eds.), Evidence-based psychotherapy: Where practice and research meet (pp. 153-177). Washington, DC, US: American Psychological Association.
Kazdin, A. E. (2007). Systematic evaluation to improve the quality of patient care: From Hope to hopeful. Pragmatic Case Studies in Psychotherapy, 3(4), 37-49. http://dx.doi.org/10.14713/pcsp.v3i4.911

Lakeman, R. (2004). Standardized routine outcome measurement: Pot holes in the road to recovery. International Journal of Mental Health Nursing, 13(4), 210215. http://dx.doi.org/ 10.1111/j.14458330.2004.00336.x

Lyon, A. R., Dorsey, S., Pullmann, M., Silbaugh-Cowdin, J. et Berliner, L. (2015). Clinician use of standardized assessments following a common elements psychotherapy training and consultation program. Administration and Policy in Mental Health, 42, 47-60. http://dx.doi.org/10.1007/s10488-0140543-7

Maden, A. (2003). Standardized risk assessment: Why all the fuss? Psychiatric Bulletin, 27(6), 201-204. http://dx.doi.org/10.1192/pb.27.6.201

Martin, A.-M., Fishman, R. et Baxter, L. (2011). Practitioners' attitudes towards the use of standardized diagnostic assessment in routine practice: A qualitative study in two Child and Adolescent Mental Health Services. Clinical Child Psychology and Psychiatry, 16(3), 407-420. http://dx.doi. org/10.1177/ 1359104510366284

Mash, E. J. et Hunsley, J. (2005). EvidenceBased Assessment of Child and Adolescent Disorders: Issues and Challenges. Journal of Clinical Child and Adolescent Psychology, 34(3), 362-379. http://dx.doi.org/ 10.1207/ s15374424jccp3403_1

McHugh, R. K. et Barlow, D. H. (2010). The dissemination and implementation of evidence-based psychological treatments. A review of current efforts. American Psychologist, 65, 73-84. http://dx.doi.org/10.1037/a0018121

McKay, R. etCoombs, T. (2012). Anexploration of the ability of routine outcome measurement to represent clinically meaningful information regarding individual consumers. Australasian Psychiatry, 20(5), 433-437. http://dx.doi. org/10.1177/1039856212458813 
McKay, R., Coombs, T. et Pirkis, J. (2012). A framework for exploring the potential of routine outcome measurement to improve mental health care. Australasian Psychiatry, 20(2), 127-133

Meehan, T., McCombes, S., Hatzipetrou, L. et Catchpoole, R. (2006). Introduction of routine outcome measures: Staff reactions and issues for consideration. Journal of Psychiatric and Mental Health Nursing, 13(5), 581-587. http://dx.doi. org/10.1111/j.1365-2850.2006.00985.x

Mellor-Clark, J., Cross, S., Macdonald, J. et Skjulsvik, T. (2016). Leading horses to water: Lessons from a decade of helping psychological therapy services use routine outcome measurement to improve practice. Administration and Policy in Mental Health and Mental Health Services Research, 43(3), 279285

Moran, P., Kelesidi, K., Guglani, S., Davidson, S. et Ford, T. (2012). What do parents and carers think about routine outcome measures and their use? A focus group study of CAMHS attenders. Clinical Child Psychology and Psychiatry, 17(1), 65-69

Pauzé, R., Cook-Darzens, S., Villeneuve, M.P., Chateauneuf, D., Petitpas, J. et Côté, J. (2017). Évaluation du fonctionnement familial : proposition d'un modèle intégratif pour soutenir la pratique clinique et la recherche, Thérapie familiale 38(3), 175-208

Reeves, K., Charter, E. et Ford, T. (2015). Measurement Issues: Is standardized diagnostic assessment feasible as an adjunct to clinical practice? A systematic review. Child and Adolescent Mental Health, 21(1), 51-63. http://dx.doi. org/10.1111/camh. 12089
Rittner, B. et Wodarski, J. S. (1995). Clinical assessment instruments in the treatment of child abuse and neglect. Early Child Development and Care, 106, 43-58. http://dx.doi.org /10.1080/0300443951060106

Valenstein, M., Goldman, B., Adler, D. A., Hackman, A., Berlant, J., Oslin, D. W., ... Sonis, W. A. (2009). Implementing standardized assessments in clinical care: Now's the time. Psychiatric Services, 60(10), 1372-1375. http:// dx.doi.org/ 10.1176/appi.ps.60.10.1372

Wodarski, J. S. (2015). Assessment methods. In J. S. Wodarski, M. J. Holosko, et M. D. Feit (Eds.), Evidence-informed assessment and practice in child welfare (pp. 99-121). Cham, Switzerland: Springer International Publishing. http:// dx.doi.org/10.1007/978-3-319-120454_7

Youn, S. J., Kraus, D. R. et Castonguay, L. G. (2012). The treatment outcome package: Facilitating practice and clinically relevant research. Psychotherapy, 49(2), 115.

Zimmerman, M. et McGlinchey, J. B. (2008). Why don't psychiatrists use scales to measure outcome when treating depressed patients? The Journal of Clinical Psychiatry, 69(12), 1916-1919. 